\title{
Immune-related IncRNAs as predictors of survival in breast cancer: a prognostic signature
}

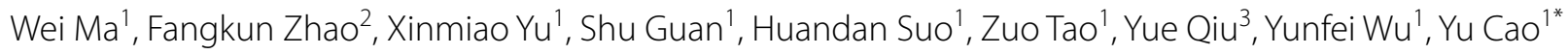
and Feng $\operatorname{Jin}^{1^{*}}$ (I)

\begin{abstract}
Background: Breast cancer is a highly heterogeneous disease, this poses challenges for classification and management. Long non-coding RNAs play acrucial role in the breast cancersdevelopment and progression, especially in tumor-related immune processes which have become the most rapidly investigated area. Therefore, we aimed at developing an immune-related IncRNA signature to improve the prognosis prediction of breast cancer.

Methods: We obtained breast cancer patient samples and corresponding clinical data from The Cancer Genome Atlas (TCGA) database. Immune-related IncRNAs were screened by co-expression analysis of immune-related genes which were downloaded from the Immunology Database and Analysis Portal (ImmPort). Clinical patient samples were randomly separated into training and testing sets. In the training set, univariate Cox regression analysis and LASSO regression were utilized to build a prognostic immune-related IncRNA signature. The signature was validated in the training set, testing set, and whole cohorts by the Kaplan-Meier log-rank test, time-dependent ROC curve analysis, principal component analysis, univariate andmultivariate Cox regression analyses.
\end{abstract}

Results: A total of 937 immune- related IncRNAs were identified, 15 candidate immune-related IncRNAs were significantly associated with overall survival (OS). Eight of these IncRNAs (OTUD6B-AS1, AL122010.1, AC136475.2, AL161646.1, AC245297.3, LINC00578, LINC01871, AP000442.2) were selected for establishment of the risk prediction model. The OS of patients in the low-risk group was higher than that of patients in the high-risk group $(p=1.215 \mathrm{e}-06$ in the training set; $p=0.0069$ in the validation set; $p=1.233 \mathrm{e}-07$ in whole cohort). The timedependent ROC curve analysis revealed that the AUCs for OS in the first, eighth, and tenth year were $0.812,0.81$, and 0.857 , respectively, in the training set, $0.615,0.68,0.655$ in the validation set, and $0.725,0.742,0.741$ in the total cohort. Multivariate Cox regression analysis indicated the model was a reliable and independent indicator for the prognosis of breast cancer in the training set $(\mathrm{HR}=1.432 ; 95 \% \mathrm{Cl} 1.204-1.702, p<0.001)$, validation set $(\mathrm{HR}=1.162 ; 95 \% \mathrm{Cl} 1.004-$ $1.345, p=0.044)$, and whole set ( $H R=1.240 ; 95 \% \mathrm{Cl} 1.128-1.362, p<0.001)$. GSEA analysis revealed a strong connection between the signature and immune-related biological processes and pathways.

Conclusions: We constructed and verified a robust signature of 8 immune-related IncRNAs for the prediction of breast cancer patient survival.

*Correspondence: caoyu@cmu.edu.cn; jinfeng@cmu.edu.cn

1 Department of Breast Surgery, The First Affiliated Hospital of China

Medical University, Shenyang, Liaoning Province, China

Full list of author information is available at the end of the article

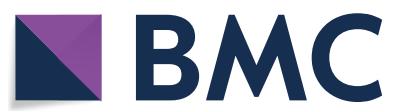

The Author(s) 2020. This article is licensed under a Creative Commons Attribution 4.0 International License, which permits use, sharing, adaptation, distribution and reproduction in any medium or format, as long as you give appropriate credit to the original author(s) and the source, provide a link to the Creative Commons licence, and indicate if changes were made. The images or other third party material in this article are included in the article's Creative Commons licence, unless indicated otherwise in a credit line to the material. If material is not included in the article's Creative Commons licence and your intended use is not permitted by statutory regulation or exceeds the permitted use, you will need to obtain permission directly from the copyright holder. To view a copy of this licence, visit http://creativeco mmons.org/licenses/by/4.0/. The Creative Commons Public Domain Dedication waiver (http://creativecommons.org/publicdomain/ zero/1.0/) applies to the data made available in this article, unless otherwise stated in a credit line to the data. 
Keywords: Breast cancer, Long non-coding RNA, Immune-related predictors, Prognostic signature, TCGA, Overall survival

\section{Background}

Among women, breast cancer is the most commonly diagnosed cancer and the leading cause of cancer-related death $[1,2]$. Globally, it is reported that nearly 2.1 million new breast cancer cases were diagnosed, and more than 0.6 million women died of breast cancer in 2018 [3]. Breast cancer is a highly heterogeneous disease and, thus, its etiology, pathological manifestations, and outcomes vary from person to person $[4,5]$. Considering the high mortality and heterogeneity, it is urgent to identify suitable detection approaches for breast cancer prognosis biomarkers. Many studies have shown that some genes and mRNAs play significant roles as prognostic molecular markers in malignancies [6-8]. Recently, research into tumor immunity has become the most rapidly advancing area within cancer. Immunotherapy provides the unprecedented opportunity to effectively treat malignancies owing to the essential involvement of the immune system in tumor development, progression, and therapy [9], especially in some malignancies such as hepatocellular carcinoma [10], early-stage squamous cell cancer of the anal canal [11], prostate cancer [12].

Long non-coding RNAs (lncRNAs) are classified as transcripts that are longer than 200 nucleotides and do not encode proteins. However, lncRNAs can physically interact with DNA, RNA, or protein. Through such interactions, lncRNAs are able to regulate gene expression at various levels such as transcriptional, post-transcriptional, and translational regulation.As a result, lncRNAs have important roles in the occurrence, progression, and prognosis of cancers and various other diseases [13-15]. In addition, recent studies have indicated that lncRNAs have crucial functions in different phases of cancer immunity such as antigen presentation, immune activation, and immune cell infiltration [16, 17]. Thus, immune-related lncRNAs have drawn considerable attention. One study has reported the prognostic merit of an immune-related lncRNA signature in the prediction of overall survival (OS) in breast cancer [5]. However, this immune-related IncRNA signature has not been validated externally by other researches yet.

In the present study, we identified and validated a robust and reliable molecular signature for the prediction of survival in breast cancer patients. Our results validated a risk scoring model based on 8 immune-related lncRNAs. The model can be used as a reliable prognostic predictor, and the 8 lncRNAs could be potential therapeutic targets for breast cancer.

\section{Methods}

Data source and preprocessing

We downloaded RNA sequencing data sets as well as the corresponding clinical characteristics of breast cancer samples and normal samples from the TCGA website (https://cancergenome.nih.gov/) in February 2020. Male breast cancer samples or samples with a follow-up time of less than 30 days were excluded. The expression levels were normalized using the Trimmed Mean of M-values method from the "edge" $\mathrm{R}$ package and underwent a $\log 2(x+1)$ transformation. R3.6.2 software was applied to normalize, process, and analyze the data. Perl (https:// www.perl.org/) was used to integrate the RNAseq value of each sample into a matrix file. A list of immune-related genes was downloaded from the gene list resources in Immunology Database and Analysis Portal (ImmPort), an open repositories of subject-level human immunology database for translational and clinical research (https:// www.immport.org/) [18].

\section{Identification of immune-related IncRNAs}

Immune-related lncRNAs were screened by co-expression analysis of immune-related genes from the list as described above. All samples were randomly split into training and validation sets using the $\mathrm{R}$ software, at a 1:1 ratio. Univariate Cox proportional hazard regression analysis was conducted to screen the immune-related lncRNAs significantly associated with overall survival (OS) in the training cohort with $p<0.01$ as the criterium. The least absolute shrinkage and selectionoperator (LASSO) regression method was used for the identification of immune-related lncRNAs most correlated with overall survival using the "glmnet" package for R.

\section{Construction of the immune-related risk prognostic system} Multivariate Cox regression analysis was used to identify significant lncRNAs for construction of the prognostic signature. We then calculated the risk score based on the expression levels of lncRNAs for each patient through following formula [19]: Riskscore $=\exp 1 * \beta 1+\exp 2 * \beta 2$ $\ldots+\operatorname{expi}^{*} \beta \mathrm{i}$ (expi was the expression value of each lncRNA, and $\beta i$ was the regression coefficient of the multivariate Cox analysis for the target lncRNA). According to the median risk score in the training set, breast cancer patients were divided into a high-risk group and a lowrisk group. 


\section{Application and validation of the risk scoring system}

In order to validate the predictive value of the model, we performed the Kaplan-Meier log-rank test, timedependent ROC curve analysis, univariate analysis, and multivariate Cox regression analysis for comparison of the survival between the high- and low-risk group in the training, validation, and the total cohort using the $\mathrm{R}$ packages "survival" and "survivalROC". We then analyzed the correlation between the expression of the 8 immunerelated lncRNAs and clinicopathological characteristics.

\section{Gene set enrichment analysis}

In order to identify different functional phenotypes between the high-risk and low-risk groups, we performed a Gene set enrichment analysis (GSEA) 4.0.3(https:// www.broadinstitute.org/gsea/index.jsp) [20]. The mRNA expression profiles of breast cancer samples from the TCGA dataset, which were divided into two groups according to risk score, were performed on GO gene sets. The study included 1000 random sample permutations and enriched gene sets with a nominal $p<0.05$ and FDR $<0.25$ were considered statistically significant. All other parameters were set based on their default values.

\section{Results}

\section{Data source and processing}

Initially, we obtained a total of 14143 lncRNA expression and 19659 gene expression profiles from 1053 breast cancer samples and 111 normal samples. In addition, the corresponding clinical data of 986 patients were downloaded from TCGA. The immune-related gene list downloaded from the ImmPort database contained 1534 immune related genes (Additional file 1: Table S1). We then obtained 937 immune-related lncRNAs through coexpression analysis of the immune gene list $(p<0.001)$. The top 10 positively and negatively correlated lncRNAs are shown sorted by correlation coefficient in Table 1.

\section{Identification of immune-related IncRNAs and construction of the prognostic model}

The data of breast cancer patients was allocated randomly to the training and validation cohort, 494 patient samples in the training cohort, 492 patient samples in the validation cohort. We carried out univariate Cox regression analysis on the expression profiles of the lncRNAs in the training set and obtained 15 candidate immune-related lncRNAs, significantly associated with survival, $p<0.01$ (Fig. 1a, Table 2). We performed Lasso regression on these lncRNAs. In order to avoid over fitting of the predicted signal, the prediction accuracy wasestimated by tenfold cross-validation (Fig. 1b, c). A total of 8 immune-related lncRNAs were obtained, including OTUD6B-AS1(HR $=1.698$; 95\% CI 1.066-2.707, $p=0.026)$ AL122010.1 $(\mathrm{HR}=0.404 ; 95 \%$

\begin{tabular}{lllll}
$\begin{array}{l}\text { Table 1 Top } \\
\text { IncRNA } \mathbf{~}^{\mathbf{1}}\end{array}$ & $\mathbf{1 0}$ & positive/negative & immune-related \\
\hline immuneGene & IncRNA & $\begin{array}{l}\text { correlation } \\
\text { coefficient }\end{array}$ & $\boldsymbol{p}$ value & Regulation \\
\hline CD19 & AC243960.1 & 0.931523591 & 0 & Positive \\
CD79B & AC243960.1 & 0.921700762 & 0 & Positive \\
TNFRSF13C & LINC00926 & 0.91156984 & 0 & Positive \\
CD3D & AC004585.1 & 0.906199227 & 0 & Positive \\
CD19 & LINC00926 & 0.902280178 & 0 & Positive \\
LCK & AC004585.1 & 0.901867543 & 0 & Positive \\
PTPRC & AL365361.1 & 0.892830584 & 0 & Positive \\
ZAP70 & AC243960.1 & 0.892093262 & 0 & Positive \\
CD3E & AC004585.1 & 0.889534261 & 0 & Positive \\
CD48 & LINC01857 & 0.888856061 & 0 & Positive \\
UBXN1 & OIP5-AS1 & -0.4915959 & $3.67 E-65$ & Negative \\
NFKBIB & OIP5-AS1 & -0.476266029 & $1.00 E-60$ & Negative \\
NFATC3 & SPINT1-AS1 & -0.468085147 & $1.89 E-58$ & Negative \\
NCK2 & AC008771.1 & -0.467026674 & $3.69 E-58$ & Negative \\
IGF2R & AC073896.4 & -0.46300298 & $4.58 E-57$ & Negative \\
UBR1 & AP001505.1 & -0.449374123 & $1.81 \mathrm{E}-53$ & Negative \\
CBL & SPINT1-AS1 & -0.448571697 & $2.91 E-53$ & Negative \\
PSMC3 & AL122035.1 & -0.448372597 & $3.28 E-53$ & Negative \\
IFNAR2 & AC008771.1 & -0.446906674 & $7.78 E-53$ & Negative \\
HSPA8 & AC108673.3 & -0.443217552 & $6.75 E-52$ & Negative \\
\hline I & & & &
\end{tabular}

1 represents sorted by correlation coefficient

CI 0.209-0.782, $p=0.007), \mathrm{AC} 136475.2(\mathrm{HR}=0.596 ; 95 \%$ CI $0.369-0.964, p=0.035)$, AL161646.1(HR $=1.215 ; 95 \%$ CI $0.954-1.549, p=0.115), \quad \mathrm{AC} 245297.3(\mathrm{HR}=0.710 ; 95 \%$ CI $0.450-1.121, p=0.142)$, LINC00578(HR $=1.269 ; 95 \%$ CI $1.001-1.609, p=0.049)$, LINC01871(HR $=0.657 ; 95 \%$ CI 0.448-0.964, $p=0.032)$, AP000442.2 ( $\mathrm{HR}=0.335$; $95 \%$ CI $0.101-1.115, p=0.075$ ) (Fig. 1d, Table 3). So OTUD6BAS1, AL161646.1 and LINC00578 were risk factors with HR > 1, while AL122010.1, AC136475.2, AC245297.3, LINC01871 and AP000442.2 were protective factors with an $H R<1$. The expression of all the 8 immune-related lncRNAs in breast cancer was shown in the Additional file 2: Figure S1 and Additional file 3: Table S2, then we compared their expression between cancer samples and normal samples (Additional file 4: Figure S2). Except for AL161646.1 and LINC00578 $(p<0.0001)$, IncRNAs, including OTUD6B-AS1, AC245297.3, AC136475.2, AL122010.1, AL161646.1 and LINC01871, were high expressed between breast cancers and normal tissues. Some of the correlation between the lncRNAs and immune genes were shown in Additional file 5: Figure S3. The risk score for each sample was calculated based on the expression levels of these 8 lncRNAs. Risk score $=\left(0.53^{*}\right.$ OTUD6B-AS1 $)+\left(-0.91^{*}\right.$ AL122010.1 $)+\left(-0.52^{*}\right.$ $\mathrm{AC} 136475.2)+\left(0.20^{*}\right.$ 


\section{a}

\begin{tabular}{|c|c|c|c|}
\hline & pvalue & Hazard ratio & $\mathbf{I}$ \\
\hline OTUD6B-AS1 & $<0.001$ & $2.153(1.373-3.376)$ & \\
\hline SNHG10 & 0.009 & $0.465(0.262-0.824)$ & $1+1$ \\
\hline AC010226.1 & 0.007 & $0.243(0.087-0.678)$ & $|-1|$ \\
\hline AL122010.1 & $<0.001$ & $0.331(0.175-0.626)$ & \\
\hline AC136475.2 & 0.002 & $0.474(0.292-0.769)$ & \\
\hline AL161646.1 & 0.002 & $1.409(1.133-1.753)$ & \\
\hline TOLLIP-AS1 & 0.005 & $0.476(0.283-0.798)$ & \\
\hline ST7-AS1 & 0.002 & $0.276(0.123-0.619)$ & 14 \\
\hline FLJ42351 & 0.008 & $0.293(0.119-0.726)$ & $\mid-t_{1}$ \\
\hline AC245297.3 & $<0.001$ & $0.470(0.301-0.733)$ & (H) \\
\hline Z68871.1 & 0.006 & $2.554(1.309-4.982)$ & \\
\hline LINC00578 & 0.007 & $1.358(1.086-1.698)$ & \\
\hline LINC01871 & 0.007 & $0.604(0.418-0.873)$ & in \\
\hline AP000442.2 & 0.004 & $0.169(0.050-0.565)$ & {$[+1$} \\
\hline AC147651.3 & 0.006 & $0.357(0.172-0.739)$ & \\
\hline
\end{tabular}

b

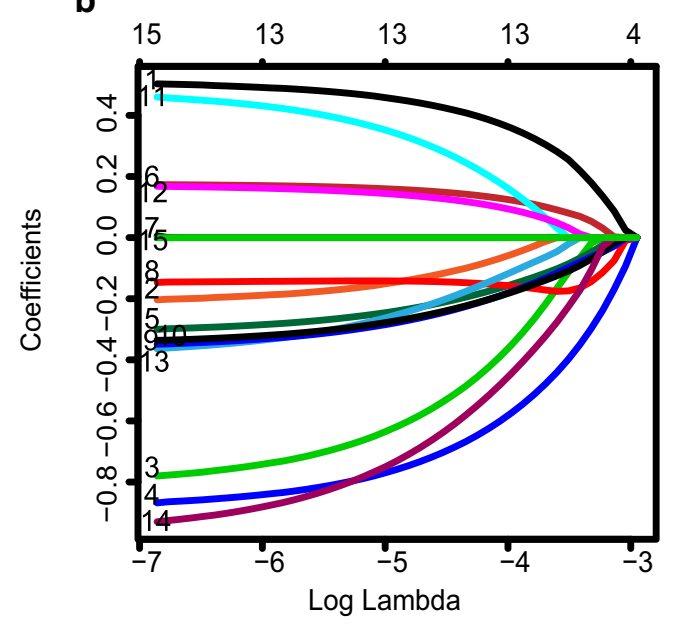

C

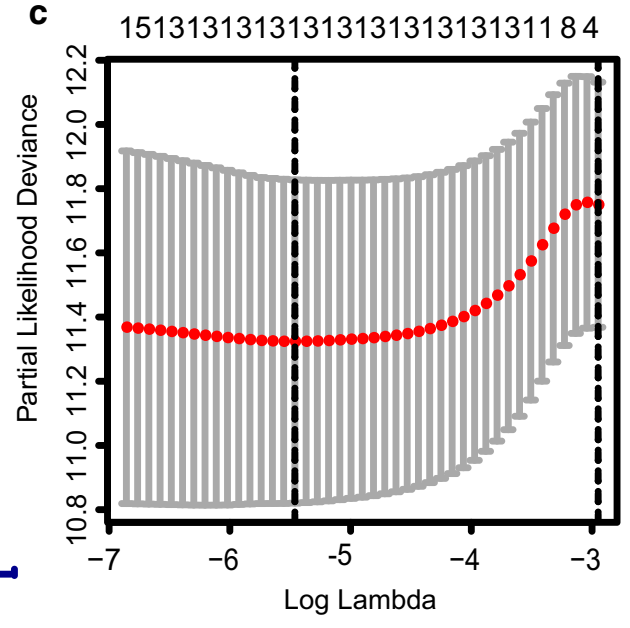

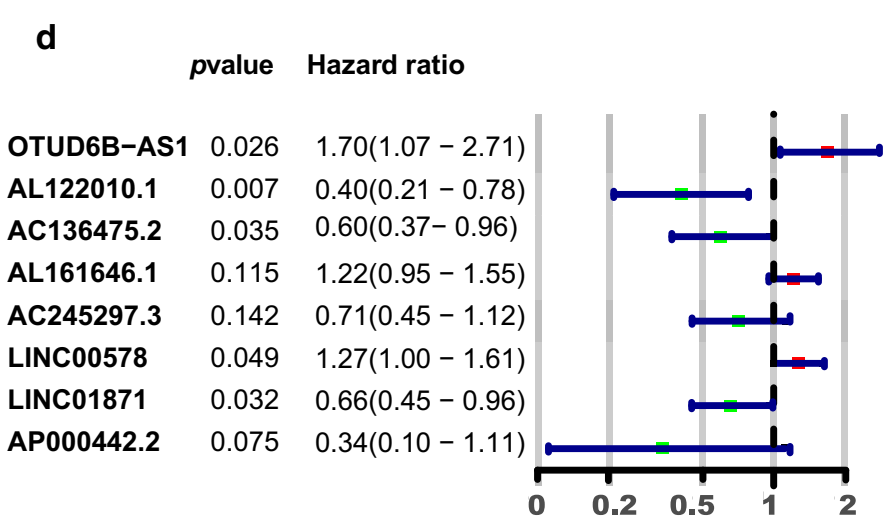

Fig. 1 Identification and construction of the immune-related IncRNAs prognostic model by univariate Cox regression and Lasso regression analysis. a Forest plot of 15 candidate immune-related IncRNAs selected by univariate Cox regression analysis associated with breast cancer survival in the training set. $\mathbf{b}$ LASSO coefficient profiles of the 15 candidates in the training set. c A coefficient profile plot was generated against the log (lambda) sequence. Selection of the optimal parameter (lambda) in the LASSO model. d Forest plot of 8 candidate immune-related IncRNAs Selected by LASSO regression analysis associated with breast cancer survival and construction prognostic model

$\mathrm{AC} 245297.3)+(0.24 *$

LINC00578) $+(-0.42 \%$ LINC01871) + (-1.10* AP000442.2) (Table 3).
The immune-related IncRNA model is a robust prognostic tool for breast cancer

Breast cancer patients were divided into low- and high-risk groups according to the median risk score in the training set. Figure $2 \mathrm{a}$ presents the result of the 
Table 2 Univariate Cox analysis for overall survival of 15 immune-related LncRNAs in training set

\begin{tabular}{lllll}
\hline ID & HR & HR.95L & HR.95H & pvalue \\
\hline OTUD6B-AS1 & 2.153122116 & 1.373184702 & 3.376046091 & 0.000832119 \\
SNHG10 & 0.464651236 & 0.261933882 & 0.824256754 & 0.008771445 \\
AC010226.1 & 0.243245961 & 0.087245203 & 0.678187397 & 0.00688694 \\
AL122010.1 & 0.330788811 & 0.174928898 & 0.625518362 & 0.000665713 \\
AC136475.2 & 0.473639558 & 0.291879867 & 0.768584807 & 0.002481537 \\
AL161646.1 & 1.409383671 & 1.132997857 & 1.753191605 & 0.00206214 \\
TOLLIP-AS1 & 0.475597922 & 0.283403961 & 0.798130634 & 0.004898673 \\
ST7-AS1 & 0.276461931 & 0.123420958 & 0.619272451 & 0.001780562 \\
FLJ42351 & 0.293301101 & 0.118560825 & 0.725581453 & 0.007952261 \\
AC245297.3 & 0.46968286 & 0.300849229 & 0.733264265 & 0.000884015 \\
Z68871.1 & 2.553691945 & 1.308915791 & 4.982247593 & 0.005970224 \\
LINC00578 & 1.358183537 & 1.086209076 & 1.698257324 & 0.007246749 \\
LINC01871 & 0.604154147 & 0.418198337 & 0.87279695 & 0.007256751 \\
AP000442.2 & 0.168810321 & 0.050411344 & 0.565287931 & 0.003913707 \\
AC147651.3 & 0.356585393 & 0.172081652 & 0.738911677 & 0.005538818 \\
\hline
\end{tabular}

Kaplan-Meier test. The $p$ value of the log-rank test was $1.215 \mathrm{e}-06$, indicating that patients in the low-risk group had a 10 year longer median OS compared with the high-risk group. To assess the accuracy of the prognostic model, further examinations in both the testing set and the whole set were performed with the same algorithm cutoff. Both sets yielded similar results. The low-risk group exhibited remarkably better overall survival (OS) than the high-risk group, which indicated that the prognostic signature was effective $(p=0.0069$ in the validation set; $p=1.233 \mathrm{e}-07$ in the total set) (Fig. 2b, c).

The ROC curve analysis of the model in the training set demonstrated its promising predictive value for breast cancer survival (1-year AUC $=0.812,5$-year $\mathrm{AUC}=0.772$, 8-year $\mathrm{AUC}=0.81,10$-year $\mathrm{AUC}=0.857$, Fig. 2d). We then validated the model in the testing set, and the 1-year AUC was 0.615, the 5-year AUC was 0.599, the 8-year AUC was 0.68, and the 10-year AUC was 0.655 (Fig. 2e). As for the total cohort, the 1-year
AUC was 0.725, the 5-year AUC was 0.678, the 8-year AUC was 0.742, and the 10-year AUC was 0.741 (Fig. 2f).

Principal component analysis (PCA) of the training, testing, and total breast cancer cohort demonstrated a different distribution pattern between the high- and lowrisk groups, based on the expression of the 8 immunerelated IncRNAs. This was indicative of the difference between the immune phenotypes of the groups (Additional file 6: Figure S4).

\section{Assessment of the correlation between candidate IncRNAs and clinicopathological characteristics}

We generated risk curves and scatter plots to display the risk score and survival status of each breast cancer patient, not only in the training set, but also in the testing and in the total set. The risk coefficient and mortality in the low-risk group were lower than those in the high-risk group (Fig. 3a-f).

Tumors with high prognostic scores expressed highrisk immune-related lncRNAs, whereas tumors with low prognostic scores expressed protective immune-related IncRNAs. The heatmap revealed that OTUD6B-AS1, AL161646.1, and LINC00578 were highly expressed in the low-risk group, while AC136475.2, AL122010.1, LINC01871, and AP000442.2 were highly expressed in the high-risk group (Fig. 3g-i).

Further, in the overall sample, we analyzed the relationship between the expression of the 8 candidate lncRNAs and different clinicopathological factors (such as T, N, M, stage of 7th AJCC, molecular typing, etc.). Our results confirmed that differential expression of AC136475.2 $(p<0.01)$, AL122010.1 $(p<0.001)$, AL161646.1 $(p<0.05), \quad$ LINC01871 $(p<0.01)$ could be observed among different $\mathrm{T}$ grades (Fig. 4a). The differences in expression of AC136475.2 $(p<0.05)$, AL161646.1 $(p<0.01)$, and OTUD6B - AS1 $(p<0.05)$ were statistically significant between different $\mathrm{N}$ groups (Fig. 4b). High expression of AL161646.1 $(\mathrm{p}<0.05)$ and LINC00578 $(p<0.001)$ was observed in M1 group, while

Table 3 Construction of 8 immune-related IncRNAs prognostic signature

\begin{tabular}{|c|c|c|c|c|c|}
\hline ID & Correlation coefficient & HR & HR.95L & HR.95H & $p$ value \\
\hline OTUD6B-AS1 & 0.529692531 & 1.69841002 & 1.065676476 & 2.706822063 & 0.025916888 \\
\hline AL122010.1 & -0.905807602 & 0.404215308 & 0.209034782 & 0.781640328 & 0.007098656 \\
\hline AC 136475.2 & -0.517003209 & 0.596304874 & 0.368979652 & 0.963683231 & 0.034771353 \\
\hline AL161646.1 & 0.195137911 & 1.215478602 & 0.953505844 & 1.549427559 & 0.115127418 \\
\hline AC245297.3 & -0.342477733 & 0.710008929 & 0.449757063 & 1.120855504 & 0.141510647 \\
\hline LINC00578 & 0.238157741 & 1.268909336 & 1.000738329 & 1.608942972 & 0.049292045 \\
\hline LINC01871 & -0.419944116 & 0.657083539 & 0.447993222 & 0.963761851 & 0.031647378 \\
\hline AP000442.2 & -1.093407536 & 0.335072774 & 0.100718288 & 1.114730656 & 0.074608365 \\
\hline
\end{tabular}



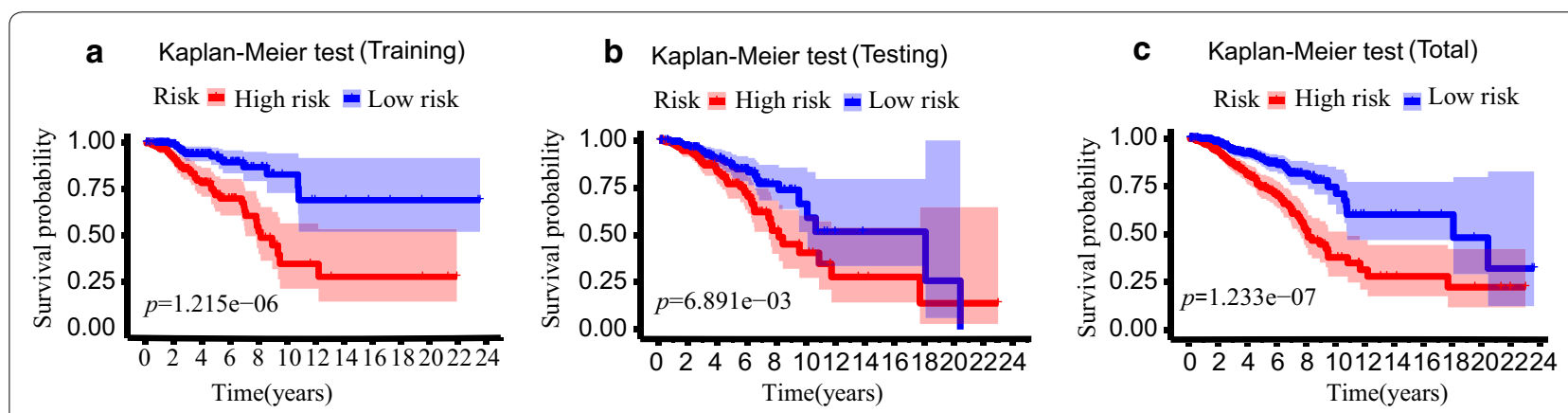

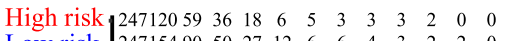

Low risk \begin{tabular}{llllllllllllllll}
247154 & 90 & 50 & 27 & 12 & 6 & 6 & 4 & 3 & 2 & 2 & 0 \\
\hline 0 & 2 & 4 & 6 & 8 & 10 & 12 & 14 & 16 & 18 & 20 & 22 & 24
\end{tabular} Time(years)

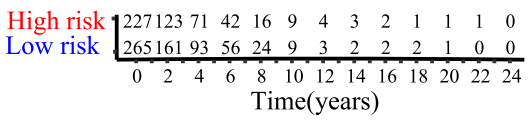

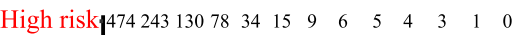
Low risk \begin{tabular}{c|ccccccccccccc}
512 & 315 & 183 & 106 & 51 & 21 & 9 & 8 & 6 & 5 & 3 & 2 & 0 \\
\hline 0 & 2 & 4 & 6 & 8 & 10 & 12 & 14 & 16 & 18 & 20 & 22 & 24
\end{tabular} Time(years)
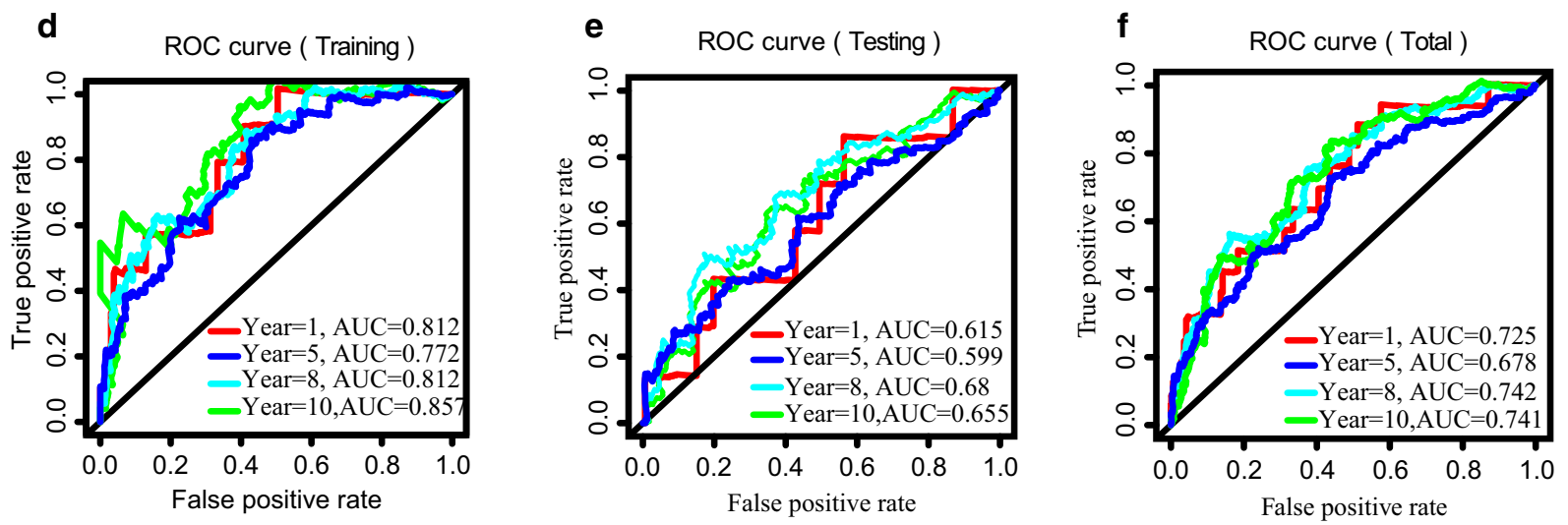

Fig. 2 Verification of immune-related IncRNAs prognostic signature's prediction ability. Kaplan-Meier survival analysis for the overall survival curves of breast cancers in the training (a), testing (b) and total set (c) with a low or high risk of death, according to the model based classifier risk score level. Time-dependent receiver operating characteristic (ROC) analysis of the sensitivity and specificity of the survival for the immune-related IncRNAs risk score in the training (d), testing (e) and total set (f)
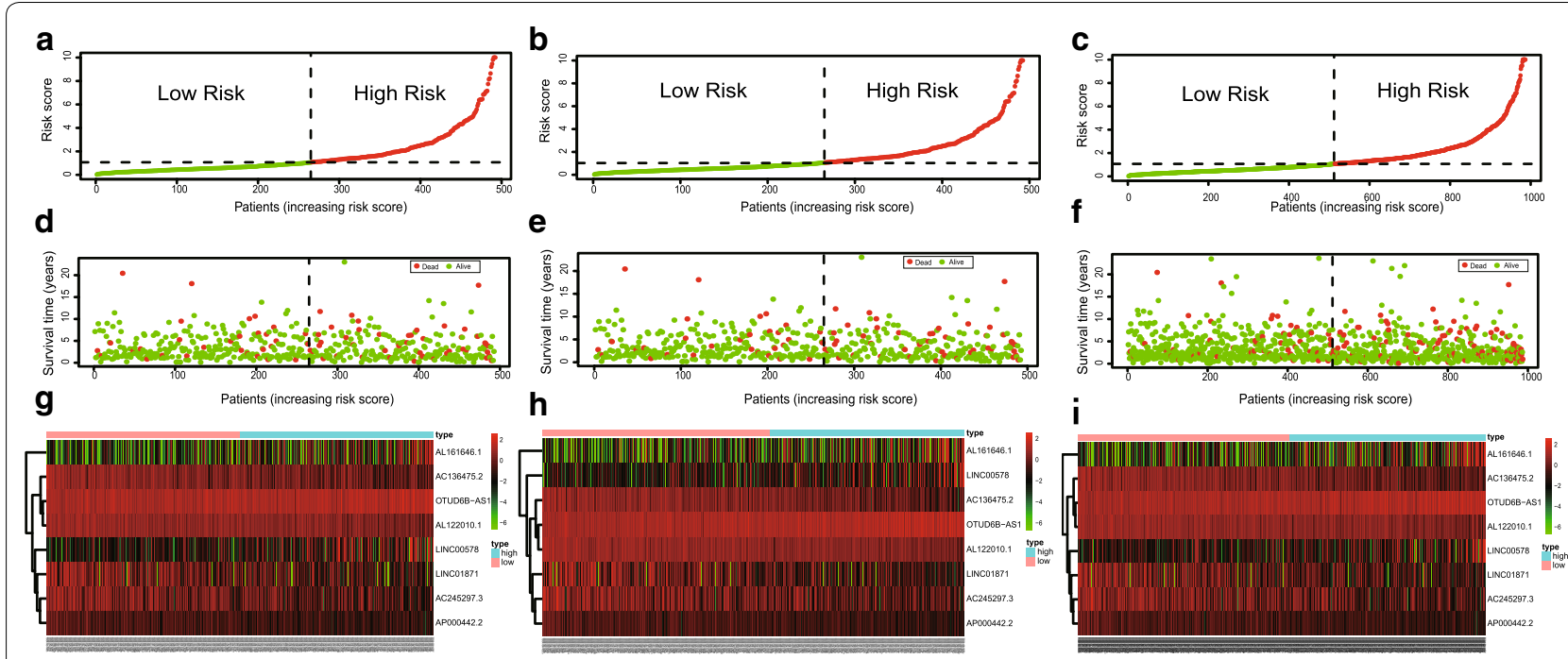

Fig. 3 Immune-related IncRNA signature risk score analysis. The signature risk score distribution in the training (a), testing (b) and total set (c). The scatter plot of the sample survival overview in the training (d), testing $(\mathbf{e})$ and total set $(\mathbf{f})$, the green and red dots respectively represent survival and death. Heatmap showed the expression profiles distribution of the signature in the low-risk groups and high-risk groups in the training (g), testing (h) and total set (i), the pink bar represented the low-risk group, and the blue bar represents the high-risk group 

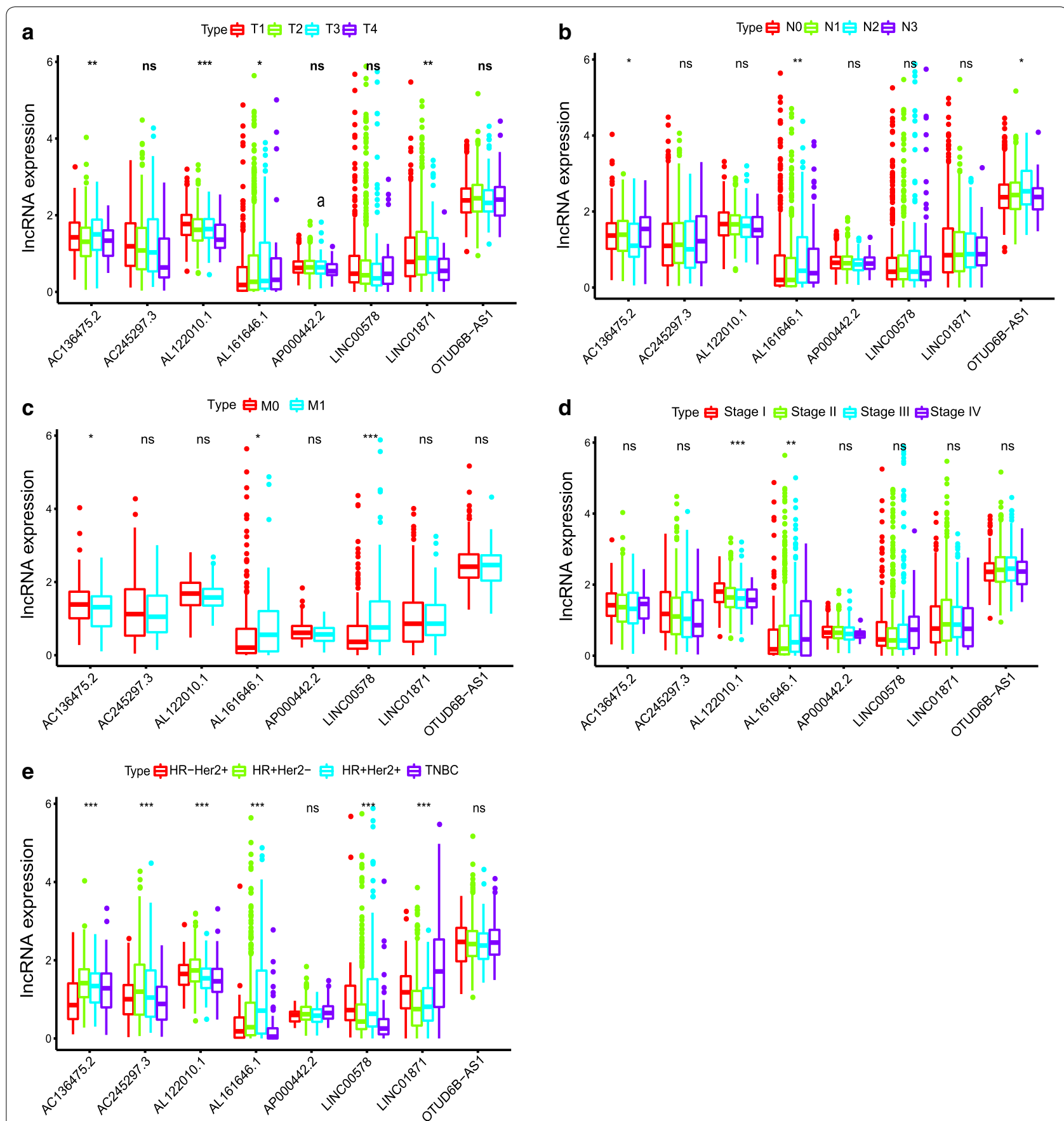

Fig. 4 Correlations between risk score of the 8 immune-related IncRNAs-based model with clinicopathological characteristics. The box-plot showed that there were statistical difference expressions of the candidate immune-related IncRNAs in T (a) N (b), molecular typing (c) in the whole cohort

low expression of AC136475.2 was observed in M1 group $(\mathrm{p}<0.05)$ (Fig. 4c). The differences in expression of AL122010.1 $(p<0.001)$ and AL161646.1 $(\mathrm{p}<0.01)$ were statistically significant between different stage groups (Fig. 4d). Except for AP000442.2 and OTUD6B-AS1,
lncRNAs, including AC136475.2, AC245297.3 $(p<0.001)$, AL122010.1 $\quad(p<0.001), \quad$ AL161646.1 $\quad(p<0.001)$, LINC00578 $(p<0.001)$, and LINC01871 $(p<0.001)$, were differently expressed between different breast cancer molecular types (Fig. 4e). 
Evaluation of the immune-related IncRNA signature as an independent prognostic factor in patients with breast cancer

We carried out univariate and multivariate Cox regression analyses to verify that the model could serve as an independent prognostic factor for breast cancer, also accounting for certain clinicopathological variables (such as age, ER status, PR status, AJCC 7th T stage, etc.) (Fig. 5). The univariate Cox analysis revealed that the high-risk group was significantly correlated with shorter survival in the training set $(\mathrm{HR}=1.483$; 95\% CI $1.273-$ $1.729, p<0.001)$, validation set $(\mathrm{HR}=1.147 ; 95 \% \mathrm{CI}$ $1.012-1.301, p=0.032)$, and whole set $(\mathrm{HR}=1.220 ; 95 \%$ CI 1.128-1.318, $p<0.001)$. Multivariate Cox regression
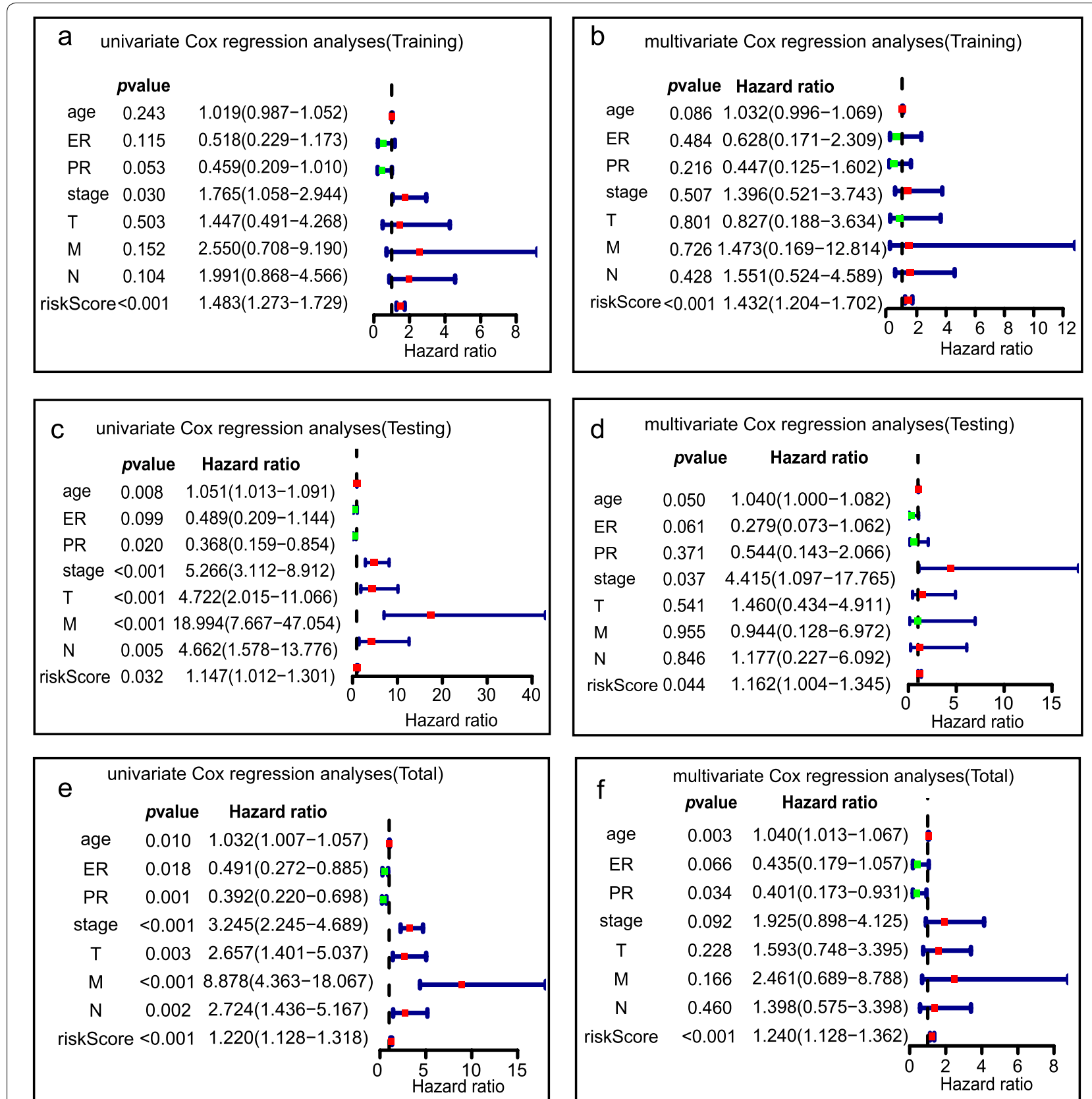

Fig. 5 The Cox regression analysis for evaluating the independent prognostic value of the risk score. Univariate (a) and multivariate Cox regression analyses (b) of the model in the training set. Univariate (c) and multivariate Cox regression analyses (d) of the model in the testing set. Univariate (e) and multivariate Cox regression analyses ( $\mathbf{f}$ ) of the model in the total set 
analyses of the above mentioned factors indicated that the immune-related lncRNA model was a reliable and independent prognostic factor for OS in the training set $(\mathrm{HR}=1.432 ; 95 \%$ CI 1.204-1.702, $p<0.001)$, validation set $(\mathrm{HR}=1.162 ; 95 \% \mathrm{CI} 1.004-1.345, p=0.044)$, and whole set $(\mathrm{HR}=1.240$; 95\% CI 1.128-1.362, $p<0.001)$. In the whole set, multivariate analysis revealed that age $(\mathrm{HR}=1.040$; 95\% CI 1.013-1.067, $p=0.003)$ and PR status $(\mathrm{HR}=0.401 ; 95 \%$ CI $0.173-0.931, p=0.034)$ were independent prognostic factors for OS.

\section{Gene set enrichment analysis for functional annotation of the immune-related risk signature}

GSEA of the risk signature was performed using the GSEA software. The results revealed that immunerelated responses were further enriched in low-risk groups compared to high-risk groups. We demonstrated 5 immune-related gene ontology terms in the GSEA results with $\mathrm{FDR}<0.25$ (Fig. 6), including the positive regulation of immune effector processes, positive regulation of the adaptive immune response, positive regulation of lymphocyte activation, regulation of $\mathrm{T}$ cell activation, and the $\mathrm{T}$ cell receptor signaling pathway.

\section{Discussion}

With the in-depth researches on lncRNAs and the immune system [21-24], scholars have realized that immune-related lncRNAs may prove to be useful not only as potential prognostic biomarkers but could also provide novel therapeutic options. Nevertheless, the lack of validation cohorts is a limitation for the proper evaluation of the prognostic merit of potential biomarkers. In the current study, we identified a novel immune-related lncRNA molecular signature using Cox and Lasso regression analyses. The signature was then validated in a testing group and a total group, indicative of its robustness and reliability. The signature demonstrated good predictive performance and could effectively classify breast cancer patients into a high-risk and a low-risk group within the training and validation sets. The low-risk group had a significantly longer overall survival compared with the high-risk group. Further, the signature proved to be an
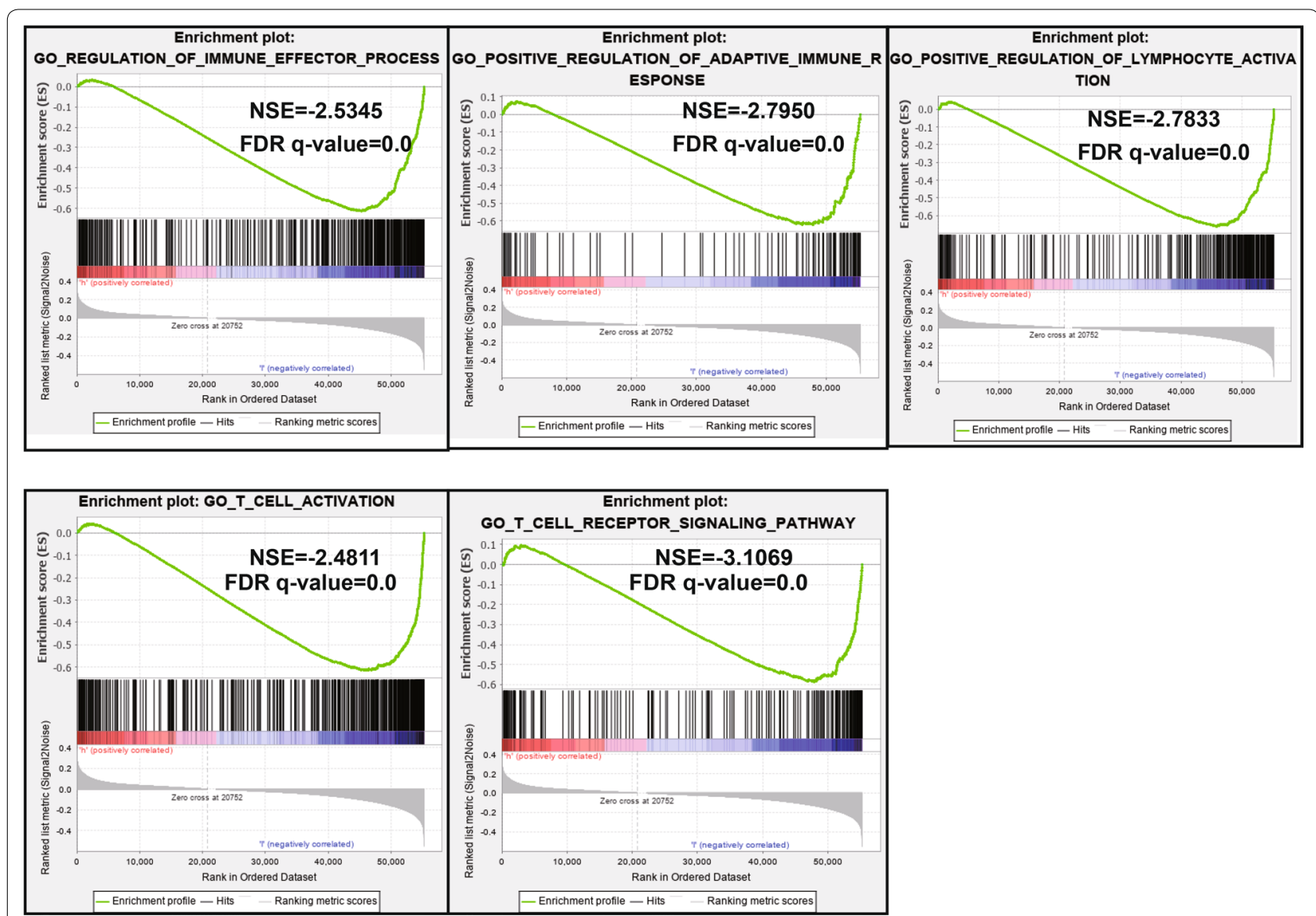

Fig. 6 GSEA analysis of the differentially expressed genes between high and low risk groups. 
independent prognostic factor based on multivariate Cox analysis, which revealed the signature's reproducibility and reliability for breast cancer prognosis.

Among the 8 candidate lncRNAs, AC136475.2, AL161646.1, LINC01871, and AP000442.2 had not been previously reported. OTUD6B-AS1 and LINC00578 were discovered as prognostic signatures in breast cancer for the first time. Although AL122010.1 and AC245297.3 had been previously reported as prognostic signatures in breast cancer [25], our research characterized their functional involvement as IncRNAs associated with tumor immunity. OTUD6B-AS1 is transcribed from the opposite strand of the OTUD6B gene, which is located on chromosome 8 in head-to-head orientation to OTUD6BAS1 [26]. It was reported that high OTUD6B-AS1 expression indicates poor prognosis in ovarian cancer [27]. However, Gang Wang et al. [28] found that high OTUD6B-AS1 expression was associated with improved survival and inhibited clear cell renal cell carcinoma proliferation via the $\mathrm{Wnt} / \beta$-catenin signaling pathway. Zhuolu Wang et al. found that OTUD6B-AS1 inhibits viability, migration, and invasion of Thyroid Carcinoma by Targeting miR-183-5p and miR-21 [29]. Further more, it was reported that the expression of miR-21 in cells of the tumor immune infiltrate, and in particular in macrophages, was responsible for promoting tumor growth [30]. Activation-induced up regulation of miR-21 biases the transcriptome of differentiating $\mathrm{T}$ cells away from memory $\mathrm{T}$ cells and toward inflammatory effector $\mathrm{T}$ cells [31]. In the current study, we found that the overall survival was lower under high OTUD6B-AS1 expression. Further, expression was higher in the N1 and N2 groups when compared to the N0 group. We speculated that OTUD6B-AS1 affects macrophages and differentiating $\mathrm{T}$ cells through miR-21, then affects immune status, and finally affects the occurrence and development of tumor. Therefore, further research and mechanistic insight are required. LINC00578 was found as a potential biomarker in lung adenocarcinoma [32, 33], major depressive disorder [34], and pancreatic cancer [35]. Although the AC243960.1 and OIP5-AS1 lncRNAs were the most positively and negatively correlated, respectively, with immune genes, they did not appear in the signature. It was indicated that high AC243960.1 expression indicates better prognosis in breast cancer, however, no statistically significant interactions between OIP5-AS1 expression and OS was found (Additional file 7: Figure S5). This may be due to the deletion of some relevant data during regression analysis.
As breast cancer is a highly heterogeneous disease, scientists divide breast cancers into different clinically relevant molecular subtypes based on the expression levels of the estrogen receptor (ER), progesterone receptor (PR), and HER2 [36-38]. Distinct prevalence, prognosis, and systemic therapies are utilized in the management of these different breast cancer subtypes [39-41]. HER2-overexpressing (ER and PR-, HER2 +) and triplenegative (ER and PR-, HER2-) subtypes are known to be more aggressive and have poorer outcomes [38, 42] Our results indicated that the expression of LINC01871 was high on both subtypes. Meanwhile, and LINC01871 shows a strong positive correlation with immune genes such as GZMB, CTLA4, PDCD1 etc. (Additional file 5: Figure S3). GZMB, the most potent cytotoxic molecules, act mainly as antitumoral and anti-infectious factors. However, when expressed by immune regulatory cells it may contribute to immune evasion of specific cancer types [43]. Cytotoxic T lymphocyte-associated antigen 4 (CTLA4) and programmed death-1 (PD-1; encoded by the PDCD1 gene) represent crucial immune checkpoints, the blockade of which can potentiate anti-tumour immunity [44, 45]. Therefore, it is suggested that LINC01871 may play an important role, particularly related to the above immune processes and immune genes, in the development of breast cancer in the two phenotypes, which requires in-depth investigation in the future.

Finally, GSEA further confirmed the robust connection of the signature with the immune response. Samples from patients with low-risk scores were associated with positive regulation of adaptive immune response. Further, patients with a high-risk score exhibited greater adaptive immune resistance. Adaptive immune resistance is a process during which cancers change their phenotype in response to a cytotoxic or pro-inflammatory immune response, thereby evading it [46]. Inhibition of adaptive immune resistance is the mechanistic foundation of responses to $\mathrm{PD}-1$ or $\mathrm{PD}-\mathrm{L} 1$ inhibition [47, 48], which has made a significant contribution to the treatment of breast cancer $[49,50]$. Our prognostic signature may provide directions for predicting the efficacy or studying the mechanism of PD-1 or PD-L1 inhibition immunotherapy. Lymphocytes, including $\mathrm{T}$ cells, $\mathrm{B}$ cells, and natural killer cells, are the main force underlying immune defense mechanisms. For them to perform their immune function, lymphocytes must be activated either through the recognition and binding of antigens or through stimulation by cytokines [51-53]. In this study, samples from patients with low-risk scores were associated with 
the positive regulation of lymphocyte activation, which may indicate that low-risk patients have a more active immune status and better immune defense than do highrisk patients. $T$ cells have been considered as having a significant role in immune surveillance and tumor eradication. On the basis of this paradigm, over the past quarter century, $\mathrm{T}$ cell-based cancer therapies have achieved success in patients $[54,55]$.

The advantage of the current study was that our signature is based on population databases and high-throughput sequencing data. Further, both exploration and validation were used in order to evaluate the risk score method. Tumor immunology has become the most rapidly advancing area of cancer research, and immunotherapy has provided promising treatment in recent years. The current study utilized a new immune-related prognostic approach for breast cancer. However, there were some limitations in this study. First, as the analyzed data was obtained from online databases, the study was of retrospective nature. Second, there is no in vitro or in vivo experimental data confirming our findings. In addition, we did not explore the potential mechanisms of investigated lncRNAs. Thus, more functional studies on the 8 lncRNAs, alone and in combination, should be carried out to further test the predictive accuracy of the signature and discover potential immune-related mechanisms. Of note, we are currently working on the clinical validation and mechanistic elucidation of these results. In various studies, gene expression differences between cancer and normal tissues are compared for the screening of prognostic genes. This may leave out certain genes with little expression differences between cancer and normal tissues. Such genes with no obvious expression difference may have a great influence on the biological behavior of tumors, chemotherapy, immunotherapy, and other factors affecting the survival of patients. Despite these limitations, to the best of our knowledge, this study is the first to report the external validation of an established immune-related lncRNA signature for breast cancer. The eight immune-related lncRNAs had never been studied in breast cancer.

\section{Conclusions}

In conclusion, we have identified an 8 immune-related lncRNA signature as a potential prognostic tool for breast cancer patients. It is strongly connected to the risk value, tumor status, and OS. The signature provides a novel insight into immune-related lncRNAs in breast cancer and identifies potential biomarkers for prognosis and immunotherapy.

\section{Supplementary information}

Supplementary information accompanies this paper at https://doi. org/10.1186/s12967-020-02522-6.

Additional file 1: Table S1. The immune-related gene list downloaded from the ImmPort database.

Additional file 2: Figure S1. The 8 immune-related IncRNAs expression in breast cancers.

Additional file 3: Table S2. The 8 immune-related IncRNAs expression in breast cancers.

Additional file 4: Figure S2. The difference expression of the 8 immunerelated IncRNAs between cancer samples and normal samples.

Additional file 5: Figure S3. The correlation between the IncRNAs and immune genes.

Additional file 6: Figure S4. Principal component analysis of the training, testing, and total set with the 8 immune-related IncRNAs signature.

Additional file 7: Figure S5. Kaplan-Meier survival analysis for the overall survival curves of breast cancers in the total set.

\section{Abbreviations}

IncRNA: Long non-coding RNA; TCGA: The Cancer Genome Atlas; OS: Overall survival; LASSO: Least absolute shrinkage and selectionoperator; ROC: Receiver operating characteristic; AUC: Area under the respective ROC; GSEA: Gene set enrichment analysis; PCA: Principal component analysis; HR: Hazard ratio; ER: Estrogen receptor; PR: Progesterone receptor; FDR: False discovery rate.

\section{Acknowledgments}

The authors would like to thank all reviewers for their valuable comments.

\section{Authors' contributions}

YC, WM and FJ designed the study. YC, ZT and HS wrote the code, WM, XY and SG analyzed data, FZ, YQ and YW wrote the manuscript. FZ and WM calculated the alternative splicing events. WM, FZ, YC and FJ revised the article according to the reviewer's opinion. All authors read and approved the final manuscript.

\section{Funding}

National Science Foundation for Young Scientists of China (Grant No.81600777)

Natural Science Foundation of Liaoning Province (Grant No. 2013225585).

China Postdoctoral Science Foundation: (Grant No. 2019M650057).

China Postdoctoral Science Foundation: (Grant No. 2017M621178).

Natural Science Foundation of Liaoning Province (Grant No.2020-MS-183).

\section{Availability of data and materials}

The datasets analysed during the current study are available in the TCGA repository (https://cancergenome.nih.gov/).

\section{Ethics approval and consent to participate}

Not applicable.

\section{Conflict of interest \\ Not applicable.}

\section{Competing interests}

The authors declare that they have no competing interests.

\section{Author details}

${ }^{1}$ Department of Breast Surgery, The First Affiliated Hospital of China Medical University, Shenyang, Liaoning Province, China. ${ }^{2}$ Department of Ophthalmology, The Fourth Affiliated Hospital of China Medical University, Shenyang, Liaoning Province, China. ${ }^{3}$ Department of Cardiovascular Ultrasound, The First 
Affiliated Hospital of China Medical University, Shenyang, Liaoning Province, China.

Received: 24 May 2020 Accepted: 8 September 2020

Published online: 23 November 2020

\section{References}

1. DeSantis CE, Ma J, Gaudet MM, Newman LA, Miller KD, Goding Sauer A, et al. Breast cancer statistics, 2019. CA Cancer J Clin. 2019;69(6):438-51.

2. Siegel RL, Miller KD, Jemal A. Cancer statistics, 2020. CA Cancer J Clin. 2020;70(1):7-30.

3. Bray F, Ferlay J, Soerjomataram I, et al. Global cancer statistics 2018: GLOBOCAN estimates of incidence and mortality worldwide for 36 cancers in 185 countries. Cancer J Clin. 2018;68:394.

4. Harbeck N, Penault-Llorca F, Cortes J, Gnant M, Houssami N, Poortmans P, et al. Breast cancer. Nat Rev Dis Primers. 2019;5(1):66.

5. Shen $Y$, Peng $X$, Shen $C$. Identification and validation of immune-related IncRNA prognostic signature for breast cancer. Genomics. 2020;1 12:2640.

6. El-Guindy DM, Wasfy RE, Abdel Ghafar MT, Ali DA, Elkady AM. Oct4 expression in gastric carcinoma: association with tumor proliferation, angiogenesis and survival. J Egypt Natl Canc Inst. 2019;31(1):3.

7. Abdel Ghafar MT, Gharib F, Abdel-Salam S, Elkhouly RA, Elshora A, Shalaby $\mathrm{KH}$, et al. Role of serum Metadherin mRNA expression in the diagnosis and prediction of survival in patients with colorectal cancer. Mol Biol Rep. 2020;47(4):2509-19.

8. Ghafar MTA, Gharib F, Al-Ashmawy GM, Mariah RA. Serum high-temperature-required protein A2: A potential biomarker for the diagnosis of breast cancer. Gene Rep. 2020. https://doi.org/10.1016/j.genre p.2020.100706

9. Fridman WH, Zitvogel L, Sautès-Fridman C, Kroemer G. The immune contexture in cancer prognosis and treatment. Nat Rev Clin Oncol. 2017;14(12):717-34.

10. Abdel Ghafar MT, Morad MA, El-Zamarany EA, Ziada D, Soliman H, Abd-Elsalam $\mathrm{S}$, et al. Autologous dendritic cells pulsed with lysate from an allogeneic hepatic cancer cell line as a treatment for patients with advanced hepatocellular carcinoma: A pilot study. Int Immunopharmacol. 2020;82:106375.

11. Phuong L, Rajdev L. Immunotherapy in Anal Cancer. Curr Oncol Rep. 2020;22(9):94

12. Yuan Z, Fernandez D, Dhillon J, Abraham-Miranda J, Awasthi S, Kim Y, et al. Proof-of-principle Phase I results of combining nivolumab with brachytherapy and external beam radiation therapy for Grade Group 5 prostate cancer: safety, feasibility, and exploratory analysis. Prostate Cancer Prostatic Dis. 2020. https://doi.org/10.1038/s41391-020-0254-y.

13. Gibb EA, Brown CJ, Wan LL. The functional role of long non-coding RNA in human carcinomas. Mol Cancer. 2011;10(1):38.

14. Sun M, Kraus WL. From discovery to function: the expanding roles of long noncoding RNAs in physiology and disease. Endocr Rev. 2015;36(1):25-64

15. Perry RB, Ulitsky I. The functions of long noncoding RNAs in development and stem cells. Development. 2016;143(21):3882-944.

16. Denaro N, Merlano MC, Lo NC. Long noncoding RNAs as regulators of cancer immunity. Mol Oncol. 2019;13(1):61-73.

17. Zhang $L, X u X$, Su X. Noncoding RNAs in cancer immunity: functions, regulatory mechanisms, and clinical application. Mol Cancer. 2020;19(1):48.

18. Bhattacharya S, Dunn P, Thomas CG, Smith B, Schaefer H, Chen J, et al. ImmPort, toward repurposing of open access immunological assay data for translational and clinical research. Sci Data. 2018;5:180015.

19. Cheng W, Ren X, Cai J, Zhang C, Li M, Wang K, et al. A five-miRNA signature with prognostic and predictive value for MGMT promoter-methylated glioblastoma patients. Oncotarget. 2015;6(30):29285-95.

20. Subramanian A, Tamayo P, Mootha VK, Mukherjee S, Ebert BL, Gillette MA, et al. Gene set enrichment analysis: a knowledge-based approach for interpreting genome-wide expression profiles. Proc Natl Acad Sci USA. 2005;102(43):15545-50.

21. Bin $X$, Hongiian $Y$, Xiping $Z$, Bo C, Shifeng $Y$, Binbin $T$. Research progresses in roles of LncRNA and its relationships with breast cancer. Cancer Cell Int. 2018:18:179.
22. Le K, Guo H, Zhang Q, Huang X, Xu M, Huang Z, et al. Gene and IncRNA co-expression network analysis reveals novel ceRNA network for triplenegative breast cancer. Sci Rep. 2019;9(1):15122.

23. Shafiee A, McGovern JA, Lahr CA, Meinert C, Moi D, Wagner F, et al. Immune system augmentation via humanization using stem/progenitor cells and bioengineering in a breast cancer model study. Int J Cancer. 2018;143(6):1470-82

24. Li B, Geng R, Wu Q, Yang Q, Sun S, Zhu S, et al. Alterations in immunerelated genes as potential marker of prognosis in breast cancer. Front Oncol. 2020;10:333

25. He Y, Li X, Meng Y, Fu S, Cui Y, Shi Y, et al. A prognostic 11 long noncoding RNA expression signature for breast invasive carcinoma. J Cell Biochem. 2019;120(10):16692-70202.

26. Takata M, Pachera E, Frank-Bertoncelj M, Kozlova A, Jüngel A, Whitfield $M L$, et al. OTUD6B-AS1 might be a novel regulator of apoptosis in systemic sclerosis. Front Immunol. 2019;10:1100.

27. Li N, Zhan X. Identification of clinical trait-related IncRNA and mRNA biomarkers with weighted gene co-expression network analysis as useful tool for personalized medicine in ovarian cancer. EPMA J. 2019;10(3):273-90

28. Wang G, Zhang ZJ, Jian WG, Liu PH, Xue W, Wang TD, et al. Novel long noncoding RNA OTUD6B-AS1 indicates poor prognosis and inhibits clear cell renal cell carcinoma proliferation via the $\mathrm{Wnt} / \beta$-catenin signaling pathway. Mol Cancer. 2019;18(1):15.

29. Wang Z, Xia F, Feng T, Jiang B, Wang W, Li X. OTUD6B-AS1 inhibits viability, migration, and invasion of thyroid carcinoma by targeting miR-183-5p and miR-21. Front Endocrinol. 2020;11:136.

30. Sahraei M, Chaube B, Liu Y, Sun J, Kaplan A, Price NL, et al. Suppressing miR-21 activity in tumor-associated macrophages promotes an antitumor immune response. J Clin Invest. 2019;129(12):5518-36.

31. Kim C, Hu B, Jadhav RR, Jin J, Zhang H, Cavanagh MM, et al. Activation of miR-21-regulated pathways in immune aging selects against signatures characteristic of memory T cells. Cell Rep. 2018;25(8):2148-62.e5.

32. Zhao B, Xu H, Ai X, Adalat Y, Tong Y, Zhang J, et al. Expression profiles of long noncoding RNAs in lung adenocarcinoma. Onco Targets Ther. 2018;11:5383-90.

33. Wang L, Zhao H, Xu Y, Li J, Deng C, Deng Y, et al. Systematic identification of lincRNA-based prognostic biomarkers by integrating lincRNA expression and copy number variation in lung adenocarcinoma. Int J Cancer. 2019;144(7):1723-34.

34. Ye N, Rao S, Du T, Hu H, Liu Z, Shen Y, et al. Intergenic variants may predispose to major depression disorder through regulation of long noncoding RNA expression. Gene. 2017;601:21-6.

35. Zhang B, Li C, Sun Z. Long non-coding RNA LINC00346, LINC00578, LINC00673, LINC00671, LINC00261, and SNHG9 are novel prognostic markers for pancreatic cancer. Am J Transl Res. 2018;10(8):2648-58.

36. Fan C, Oh DS, Wessels L, Weigelt B, Nuyten DS, Nobel AB, et al. Concordance among gene-expression-based predictors for breast cancer. N Engl J Med. 2006;355(6):560-9.

37. Hammond ME, Hayes DF, Dowsett M, Allred DC, Hagerty KL, Badve S, et al. American Society of Clinical Oncology/College Of American Pathologists guideline recommendations for immunohistochemical testing of estrogen and progesterone receptors in breast cancer. J Clin Oncol. 2010;28(16):2784-95.

38. Perou CM, Sørlie T, Eisen MB, van de Rijn M, Jeffrey SS, Rees CA, et al. Molecular portraits of human breast tumours. Nature. 2000:406(6797):747-52.

39. Howlader N, Altekruse SF, Li Cl, Chen VW, Clarke CA, Ries LA, et al. US incidence of breast cancer subtypes defined by joint hormone receptor and HER2 status. J Natl Cancer Inst. 2014. https://doi.org/10.1093/jnci/dju055.

40. Chavez-MacGregor M, Mittendorf EA, Clarke CA, Lichtensztajn DY, Hunt KK, Giordano SH. Incorporating tumor characteristics to the American joint committee on cancer breast cancer staging system. Oncologist. 2017;22(11):1292-300.

41. Waks AG, Winer EP. Breast cancer treatment: a review. JAMA. 2019;321(3):288-300

42. Yin WJ, Lu JS, Di GH, Lin YP, Zhou LH, Liu GY, et al. Clinicopathological features of the triple-negative tumors in Chinese breast cancer patients. Breast Cancer Res Treat. 2009;115(2):325-33. 
43. Arias M, Martínez-Lostao L, Santiago L, Ferrandez A, Granville DJ, Pardo J. The untold story of granzymes in oncoimmunology: novel opportunities with old acquaintances. Trends Cancer. 2017;3(6):407-22.

44. Kwek SS, Cha E, Fong L. Unmasking the immune recognition of prostate cancer with CTLA4 blockade. Nat Rev Cancer. 2012;12(4):289-97.

45. Cha JH, Chan LC, Li CW, Hsu JL, Hung MC. Mechanisms controlling PD-L1 expression in cancer. Mol Cell. 2019;76(3):359-70.

46. Ribas A. Adaptive immune resistance: how cancer protects from immune attack. Cancer Discov. 2015:5(9):915-9.

47. Taube JM, Anders RA, Young GD, Xu H, Sharma R, McMiller TL, et al. Colocalization of inflammatory response with B7-h1 expression in human melanocytic lesions supports an adaptive resistance mechanism of immune escape. Sci Transl Med. 2012;4(127):127ra37.

48. Rooney MS, Shukla SA, Wu CJ, Getz G, Hacohen N. Molecular and genetic properties of tumors associated with local immune cytolytic activity. Cell. 2015;160(1-2):48-61.

49. Li CW, Lim SO, Chung EM, Kim YS, Park AH, Yao J, et al. Eradication of triple-negative breast cancer cells by targeting glycosylated PD-L1. Cancer Cell. 2018;33(2):187-201.e10.

50. Voorwerk L, Slagter M, Horlings HM, Sikorska K, van de Vijver KK, de Maaker $\mathrm{M}$, et al. Immune induction strategies in metastatic triple-negative breast cancer to enhance the sensitivity to PD-1 blockade: the TONIC trial. Nat Med. 2019;25(6):920-8.
51. Berridge MJ. Lymphocyte activation in health and disease. Crit Rev Immunol. 2017;37(2-6):439-62.

52. Tangye SG, Pelham SJ, Deenick EK, Ma CS. Cytokine-mediated regulation of human lymphocyte development and function: insights from primary immunodeficiencies. J Immunol. 2017;199(6):1949-58.

53. Heinzel S, Marchingo JM, Horton MB, Hodgkin PD. The regulation of lymphocyte activation and proliferation. Curr Opin Immunol. 2018;51:32-8.

54. Louis CU, Savoldo B, Dotti G, Pule M, Yvon E, Myers GD, et al. Antitumor activity and long-term fate of chimeric antigen receptor-positive T cells in patients with neuroblastoma. Blood. 2011;118(23):6050-6.

55. Rosenberg SA, Yang JC, Sherry RM, Kammula US, Hughes MS, Phan GQ, et al. Durable complete responses in heavily pretreated patients with metastatic melanoma using T-cell transfer immunotherapy. Clin Cancer Res. 2011:17(13):4550-7.

\section{Publisher's Note}

Springer Nature remains neutral with regard to jurisdictional claims in published maps and institutional affiliations.
Ready to submit your research? Choose BMC and benefit from:

- fast, convenient online submission

- thorough peer review by experienced researchers in your field

- rapid publication on acceptance

- support for research data, including large and complex data types

- gold Open Access which fosters wider collaboration and increased citations

- maximum visibility for your research: over $100 \mathrm{M}$ website views per year

At BMC, research is always in progress.

Learn more biomedcentral.com/submissions 